\title{
Research on BP Neural Network Algorithm Based on Genetic Algorithm Optimization in Short-Term Power Generation Forecasting
}

\author{
Jianna Zhao \\ Department of Economics and Management \\ North China Electric Power University \\ NO.689 Huadian Road, Beishi district, Baoding, 071003, \\ China \\ azhjnzhf@163.com
}

\begin{abstract}
In order to overcome the shortcomings of traditional BP neural network, and realize the fast and accurate prediction, this paper will construct a new prediction method by combining genetic algorithm and neural network. The method significantly improves the optimization ability of the model, which can effectively overcome the slow learning speed of neural network, and overcome the blindness of the initial weights of the neural network, so as to effectively improve the accuracy of prediction. The examples show that this method can effectively improve the prediction accuracy.
\end{abstract}

Keywords-Genetic algorithm, forecasting, BP neural network

\section{INTRODUCTION}

A city's electricity consumption will change with change of the resident population and the economic growth rate. Prediction has become a hot topic in the research of power investment because of its key role in power investment decision making. Research on prediction method has experienced the development stages from a single prediction method of combination forecasting method; the prediction accuracy has been greatly improved.[1]

In recent years, with the development of artificial intelligence technology, the application of artificial neural network in the prediction of more and more widely, there are commonly used feedforward neural network (BP neural network), but BP neural network has some inherent defects, such as slow convergence, easy to fall into local optimum, the initial weights and threshold value random, the study found that the genetic algorithm is introduced to optimize the neural network, which can effectively improve the prediction accuracy. Based on this idea, this paper uses the genetic algorithm to optimize the BP neural network combination model method, and the forecast of the power generation.

\section{A BRIEF INTRODUCTION OF GENETIC ALGORITHM AND BP NEURAL NETWORK}

\section{A. Genetic Algorithm}

Genetic algorithm (GA) is a kind of parallel random search optimization method, which simulates the genetic mechanism and biological evolution in nature. [2] The nature of "survival of the fittest, the principle of biological evolution

\author{
Xiaobo He \\ Department of Economics and Management \\ North China Electric Power University \\ NO.689 Huadian Road, Beishi district, Baoding, 071003, \\ China \\ bhxb8858460@163.com
}

of municipal life" is introduced to optimize the parameters of forming the encoding series groups, according to the selection of fitness function and the genetic selection crossover and mutation of the individual selection of fitness better individual fitness is reserved, poor individuals are eliminated, the new group inherits the generation of information, and better than the previous generation. This is repeated until the condition is satisfied.

\section{B. BP neural network}

BP neural network is a kind of multilayer feedforward neural network. The network and its main characteristics are the forward transmission of signals and the error back propagation. Prior to delivery, the input signal from the input layer and hidden layer after treatment, until the output layer. [3]Each layer of neurons only affect the neurons in the next layer. If the output layer can not get the expected output, then transferred to the reverse propagation, according to the prediction error to adjust the network to be advised and threshold value, so that the BP neural network prediction output is expected to approximate the desired output. The topological structure of BP neural network is shown in Fig. 1.[4]

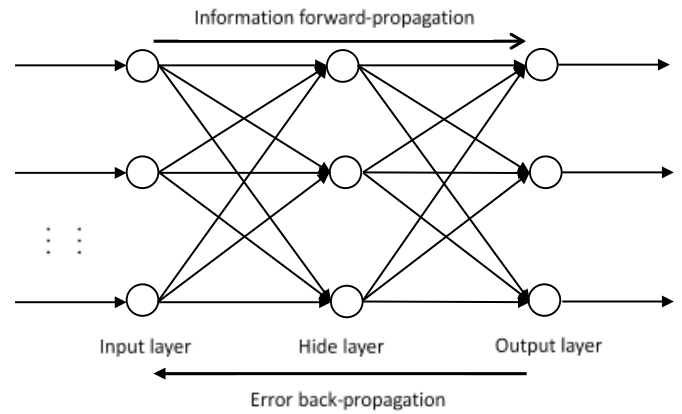

Fig. 1. Three-layer BP network model

\section{GABP MODEL BUILDING}

Genetic algorithm optimization BP neural network process as shown in Fig. 2.[5]

Genetic algorithm optimization BP neural network is divided into 3 parts: the structure of BP neural network, 
genetic algorithm optimization and BP neural network prediction.[6]Among them, the structure of BP neural network determines the structure of BP neural network according to the number of input and output parameters of the fitting function, and then determines the length of the genetic algorithm. The genetic algorithm to optimize BP neural network weights and thresholds of each individual of the population contains a network of ownership and threshold value, the individual fitness degree is calculated by value the fitness function of genetic algorithm, through selection, crossover and mutation operation to find the optimal fitness value of individual. BP neural network prediction using the genetic algorithm to get the optimal individual neural network initial weights and threshold reproduction, the network after the training to predict the output function.

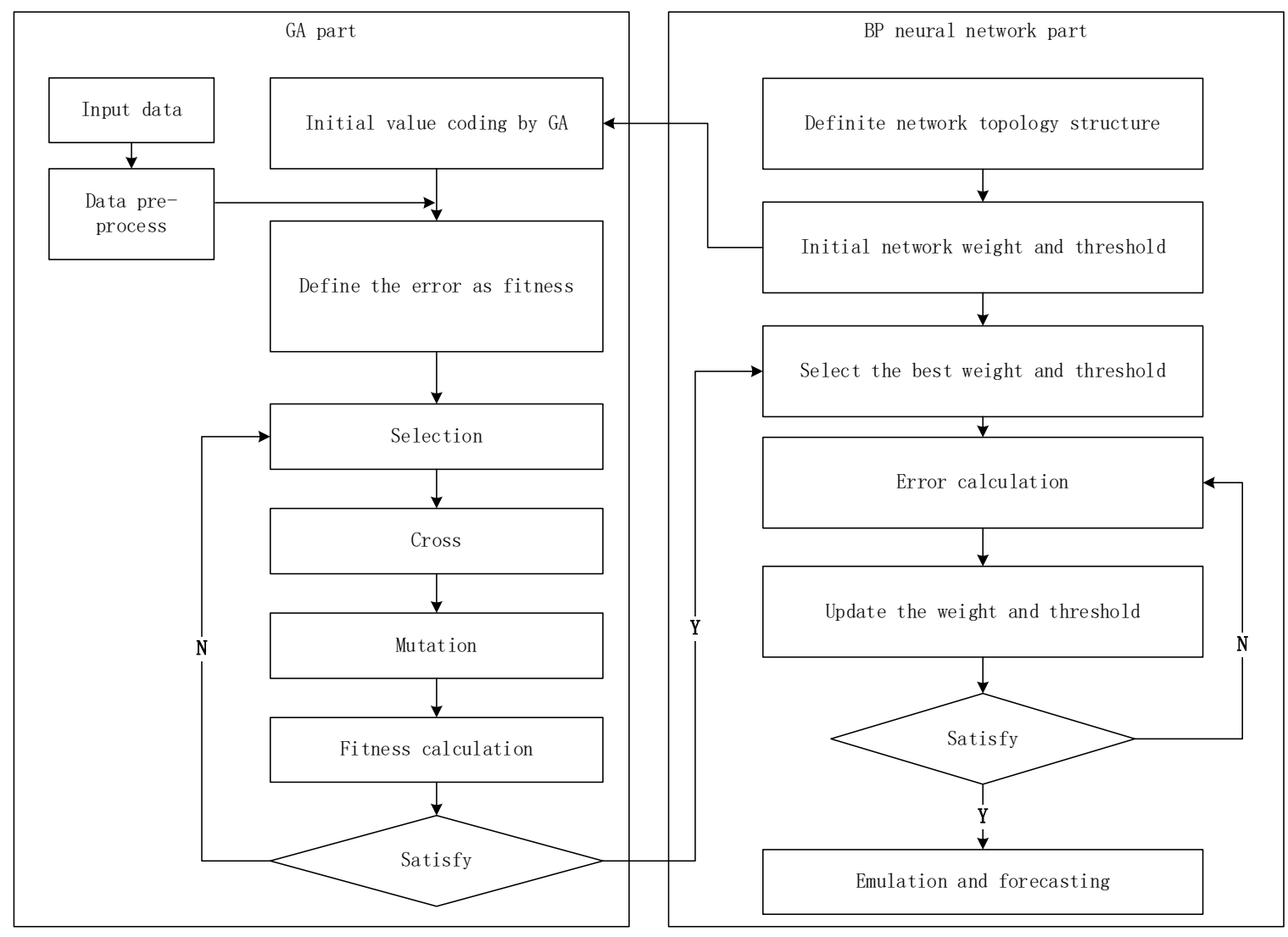

Fig. 2. Optimization of the BP neural network by GA

\section{CASE STUDY}

\section{A. Parameters Setting}

Forecast future electricity generation by data from 2000--2011 in Xi'an. Permanent population at the end of this year, production capacity, production and second industrial output value to predict the next year's electricity generation.

TABLE I. PARAMETERS OF MODELS MENTIONED IN THE PAPER

\begin{tabular}{|c|c|}
\hline Model & \multicolumn{1}{|c|}{ Parameters } \\
\hline GA & $\begin{array}{r}\text { Iterations=50, size=10, crossover rate=0.4, } \\
\text { mutation rate }=0.2\end{array}$ \\
\hline BPNN & $\begin{array}{l}\mathrm{N}_{\max }=100 ; \text { hidden layer node=[5];learning } \\
\text { rate=0.1;goal=0.00004 network } \\
\text { structure:4-5--1 }\end{array}$ \\
\hline
\end{tabular}

\section{B. Model performance evaluation}

To examine the performance of model, the mean absolute percentage error (MAPE) and the mean square error (MSE) are proposed to measure the forecast accuracy. The formulas are as follow:

$$
\begin{aligned}
& \text { MAPE }=\frac{1}{n} \sum_{i=1}^{n}\left|\frac{\hat{y}_{i}-y_{i}}{y_{i}}\right| \\
& M S E=\frac{1}{n} \sum_{i=1}^{n}\left(\hat{y}_{i}-y_{i}\right)^{2}
\end{aligned}
$$

Where yi represents the actual value at period $\mathrm{i}$; y^ is the forecasting value at period $i$ and $n$ is the number of forecasting period. 


\section{Analysis of forecasting results}

Fig. 3 shows the prediction results of GABP, Fig. 7 and Fig. 8 show the GABP curve and GABP network prediction error. Table 2 shows the predictive value of BP and GABP. Table 3 shows the prediction accuracy of GABP and BP.

In order to show the superiority of GABP, we compare the traditional BP neural network with the optimized GABP model, and table 2 shows the predicted value and the actual value of the two models. It can be seen clearly that the stability of the GABP model, there is no large fluctuations.

In order to test the validity of the model, we use MAPE and MSE two indexes to measure the accuracy of the model. Can be seen from table 3, the prediction model of GABP MAPE was 0.074287, 0.086709 lower than the BP, and GABP MSE is 55.8401, is lower than the 249.0963 of the BP. It shows that the stability of the GABP prediction model and the prediction accuracy is better than the traditional BP model, which can be used to predict the short-term power generation.

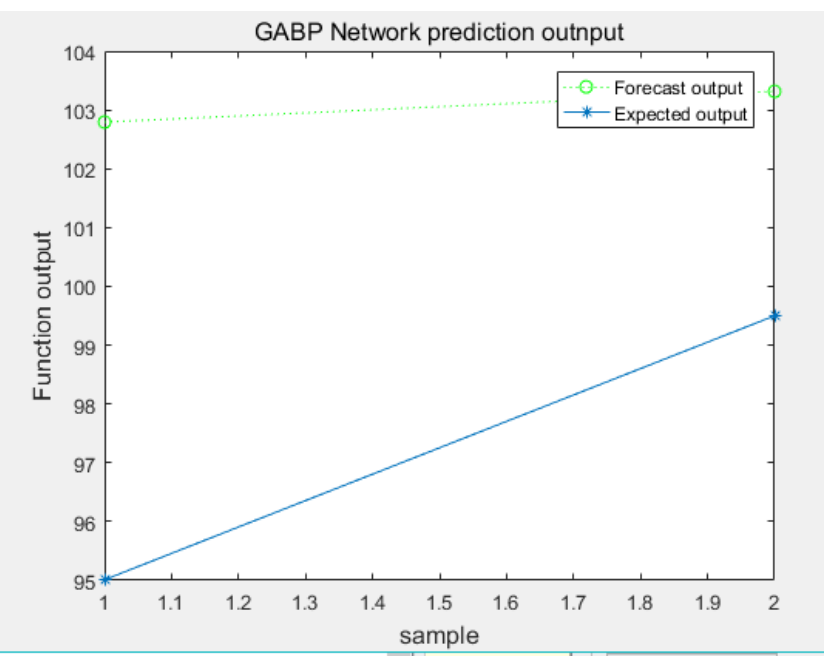

Fig. 3. Forecasting results of GABP

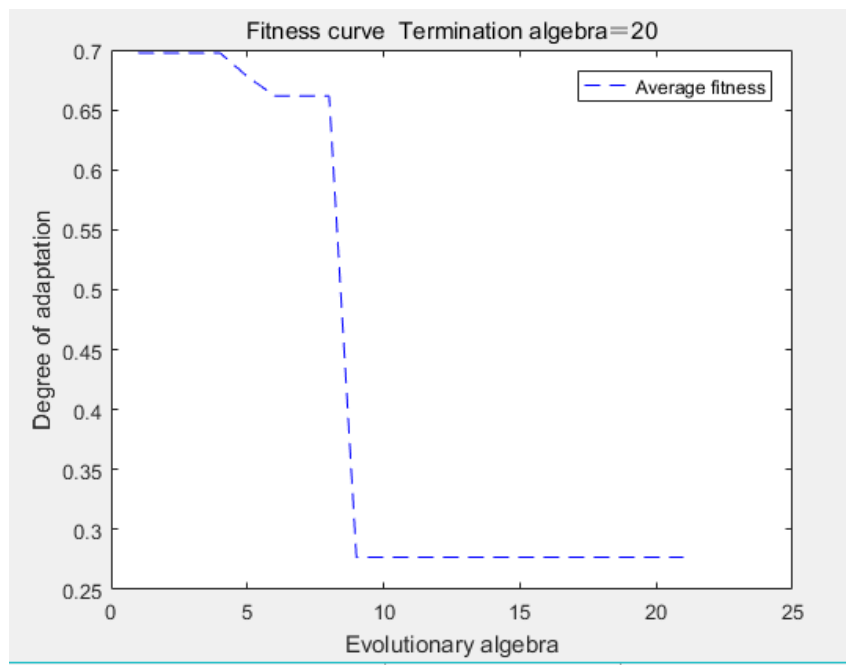

Fig. 4. Fitness curve of GABP

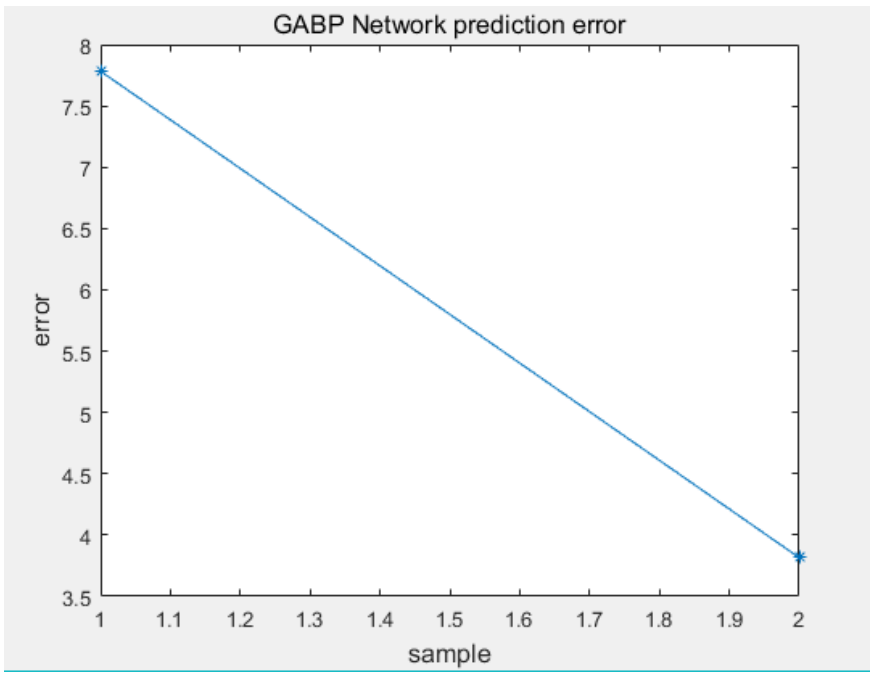

Fig. 5. Error of GABP

TABLE II. RESULTS of models

\begin{tabular}{|c|c|c|}
\hline Yodel & 2011 & 2012 \\
\hline Actual & 95.01 & 99.5 \\
\hline GABP & 104.27 & 104.57 \\
\hline BP & 116.66 & 94.07 \\
\hline
\end{tabular}

TABLE III. MODELS PERFORMANCE EVALUATIONS

\begin{tabular}{|c|c|c|}
\hline Index $\quad$ Model & GABP & $\mathrm{BP}$ \\
\hline MAPE & 0.074287 & 0.086709 \\
\hline MSE & 55.84081 & 249.0963 \\
\hline
\end{tabular}

\section{CONCLUSION}

In order to strengthen the economy of electric power investment, to avoid the waste of investment, and to improve the accuracy of power generation forecasting is of great significance.[7] Due to the short-term power generation is affected by many kinds of external factors, its existence is not regular, so it can not use a single model to forecast the power generation. According to the randomness of the traditional neural network weights and threshold, this paper proposes a prediction based on genetic algorithm BP neural network model, and compare it with the traditional BP neural network prediction results, the prediction results of MAPE and MSE is better than BP neural network has higher prediction accuracy. The GA algorithm is simple and easy to understand, easy to operate, and can be widely used in intelligent algorithm parameter optimization. In summary, the GABP prediction model has the characteristics of high precision and simple operation, which provides a scientific and effective reference for Short-Term prediction of power generation.

\section{REFERENCES}

[1] Niu Dongxiao et al., based on the combination of long term power load forecasting based on genetic algorithm, in twenty-sixth China Control Conference 2007: Zhangjiajie Hunan 790-793 page.

[2] Shi Yingling, Yang Hongsong and Pang Nansheng, an adaptive hybrid genetic optimization algorithm for Short-Term electric load forecasting. Electric power science and engineering, 2008 (09): 32-35 page. 
[3] Cheng Yugui, dawn and Lin Mingyu, based on genetic algorithm and BP neural network for the prediction and analysis of long term electric load in urban areas. Computer applications, 2010 (01): 224-226 page.

[4] Niu Dongxiao et al. Research and application of genetic neural network optimization prediction method. Journal of North China Electric Power University, 2001.28 (1).

[5] Ma Yanpeng, the application of adaptive genetic neural network in the prediction of daily water consumption, in Second China Intelligent Computing Conference 2008: China Luoyang Henan. Fourth pages.

[6] Li Lingchun and Lily Tien, based on genetic algorithm and BP neural network for Short-Term electric load forecasting. Journal of Anhui University of Technology and Science (NATURAL SCIENCE EDITION), 2009 (03): 57-60.

[7] Zhang Baoping, based on Genetic BP neural network for Short-Term load forecasting of power system, 2003, Xi'an University of technology. Sixty-ninth pages. 